\title{
RECONSTRUCCIÓN MICROQUIRÚRGICA DE LENGUA*
}

\author{
Drs. Luis Eduardo Nieto Ramírez MD. FACS ${ }^{1}$, Clara Natalia Pedraza Borrero MD²
}

1 Cirujano Plástico Microcirujano, Cirujano de Mano, Epidemiólogo Clínico. Hospital Universitario San Ignacio. Hospital Militar Central. Profesor Pontificia Universidad Javeriana. Universidad Militar Nueva Granada. Director Médico Centro Internacional de Microcirugía y Nervio Periférico.

2 Residente IV Cirugía Plástica Pontificia Universidad Javeriana.

Bogotá, Colombia.

\begin{abstract}
Microsurgical tongue reconstruction

Malignant tumors of tongue are a common pathology with high morbidity and mortality. Treatment requires surgical oncology and systemic management, with the respective reconstruction in order to achieve an adequate quality of life, due to the primary function of the tongue during feeding, communication, social and labor interaction. That is why the choice of donor tissue for reconstruction depends heavily on its characteristics and the type of defect, essential to obtain favorable results in the patients. A review of the classification of resulting defects after tongue's tumors resection is performed, and management algorithm and microvascular free flaps more frequently used in this type of reconstruction.
\end{abstract} oncology.

Key words: Tongue cancer, tongue reconstruction, reconstructive microsurgery, free flaps, surgical

\section{Resumen}

Los tumores malignos de lengua son una patología frecuente con alto grado de morbilidad y mortalidad. $\mathrm{Su}$ tratamiento requiere manejo quirúrgico y sistémico oncológico, con la respectiva reconstrucción, para lograr así una adecuada calidad de vida, debido a la función primordial de la lengua durante la alimentación, la comunicación, la interacción social y laboral. Es por esto que la elección de los tejidos donantes para ello depende en gran medida de sus características y del tipo de defecto, aspecto fundamental para obtener resultados favorables en los pacientes. Se realiza una revisión de la clasificación de los defectos resultantes después de la resección de tumores de lengua, un algoritmo de manejo y los colgajos libres microvasculares más utilizados en este tipo de reconstrucción.

Palabras clave: Cáncer de lengua, reconstrucción lingual, microcirugía reconstructiva, colgajos libres, cirugía oncológica.

*Recibido el 25 de agosto de 2015 y aceptado para publicación el 11 de octubre de 2015.

Conflictos de interés: Ninguno

Correspondencia: Dr. Luis E. Nieto R.

lenietor@microcirugiamd.com 


\section{Introducción}

El carcinoma escamocelular de orofaringe es el sexto tumor con mayor incidencia en el mundo ${ }^{1}$, el de lengua es el cáncer primario más frecuente de la cavidad oral. En los Estados Unidos, el cáncer de boca ocupa el $8 \%$ de todos los tumores, siendo los hombres más propensos que las mujeres, en especial hombres mayores de 50 años. Históricamente, los individuos de raza negra tienen una doble incidencia por encima de individuos de raza blanca y en un $75 \%$ de los casos son individuos que consumen grandes cantidades de alcohol y tabaco. En Asia la incidencia es significativamente mayor debido al consumo de nuez de betel, cigarrillo y alcohol ${ }^{2}$. En Colombia el cáncer es la tercera causa de muerte y el carcinoma escamocelular de boca ocupa el quinto lugar entre todos los cánceres, con una relación hombre/mujer 2:1. Al año se presentan aproximadamente 2.000 nuevos casos de cáncer oral, en su mayoría diagnosticados en la población mayor de 60 años y de forma escasa en menores de 40 años, estos casos están asociados a factores de riesgo como el tabaco ${ }^{3}$.

El carcinoma de cavidad oral no responde bien a quimioterapia y radioterapia, por lo cual es necesario, para un adecuado tratamiento, márgenes de resección amplios, conduciendo a los pacientes a la glosectomía como parte del mismo, los que requieren posteriormente una reconstrucción adecuada.

Los objetivos de la reconstrucción lingual son, primero, la obliteración de la cavidad oral, entendiéndose esto como el contacto que deben hacer las diferentes superficies mucosas cuando la boca está cerrada; segundo, el contacto de la lengua reconstruida con el paladar y la premaxila para lograr adecuada articulación de sonidos; tercero, mantener la movilización de la saliva y, por último, recuperar la sensibilidad ${ }^{4}$.

Para lograr los objetivos anteriormente mencionados se deben tener en cuenta principios como: sobrecorregir el volumen del defecto, reconstruir el piso de la boca con tejidos donantes delgados y el tejido lingual con tejidos gruesos, la protrusión de la lengua reconstruida y la restauración del volumen de los compartimentos adyacentes a la lengua, sin bloquear los surcos glosoalveolar, bucoalveolar y labioalveolar, facilitando así la movilización de secreciones.

\section{Clasificación de los defectos linguales}

La clasificación de los defectos linguales está basada en su desarrollo embriológico por sus dos planos de fusión, el de línea media que define la longitud de la lengua y el coronal que la divide en dos tercios anteriores y un tercio posterior. Este último es considerado como parte de la orofaringe, la cual debe ser reconstituida de la mejor forma manteniendo en lo posible intacta la función neurológica del esfínter (Figura 1). Los dos tercios anteriores de la lengua participan en la fase preparatoria de la deglución y forman el bolo alimenticio para la fase faríngea, además de las funciones digitales de la lengua como el barrido de los alimentos de los surcos labial y bucal, la limpieza labial y hacer parte de la competencia oral.

Según la resección realizada, el defecto se puede clasificar en 5 tipos $^{5}$ :

Tipo 1. Hemiglosectomía subtotal. También denominada resección local ampliada que incluye la resección de un tercio o menos del volumen total de la lengua (Figura 2).

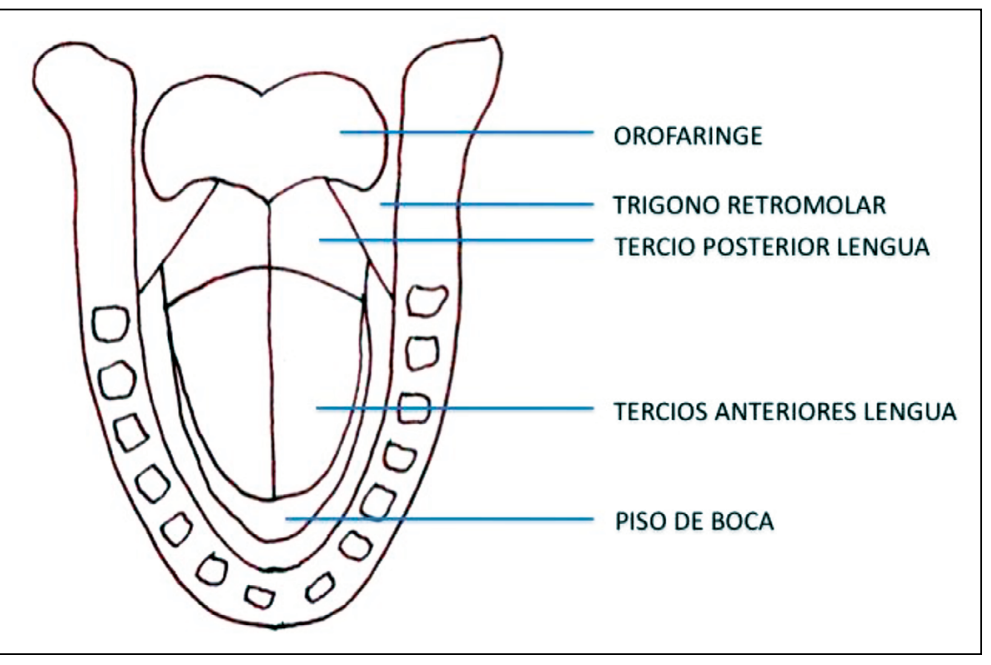

Figura 1. Áreas de la cavidad oral más importantes para tener en cuenta en la clasificación de los defectos linguales. 
Tipo 2. Hemiglosectomía. Incluye la mitad del piso de la boca, la mitad de la lengua y puede comprometer la base de la misma (Figura 3).

Tipo 3. Hemiglosectomía extendida. La extensión incluye parte del piso anterior de la boca contralateral, y/o pilar amigdalino y/o rafe lingual (Figura 4).

Tipo 4. Glosectomía subtotal. El defecto incluye todo el piso de la boca, los dos tercios anteriores de la lengua y puede incluir la mitad de la base de la lengua (Figura 5).

Tipo 5. Glosectomía total. Incluye la totalidad de la lengua y el piso de la boca (Figura 6).

\section{Formas de reconstrucción}

Dentro de las múltiples opciones quirúrgicas el método reconstructivo escogido depende de factores como la cantidad de tejido lingual postresección, la posibilidad de protrusión de este remanente, el estado de tejidos adyacentes y las condiciones generales de cada paciente (Figura 7).

Para reconstruir defectos Tipo 1, hemiglosectomías subtotales, colgajos locales como el melolabial o el mucoso-bucal son buena opción, especialmente en pacientes de tercera edad debido a la laxitud propia de los tejidos, así como también el avance de la base de la lengua en defectos laterales ${ }^{6}$ y la rotación de los colgajos remanentes de la misma lengua. Siempre se debe tener en cuenta cumplir la condición reconstructiva de proveer adecuada obliteración de la cavidad oral y la protrusión de la lengua.

En defectos Tipo 2 y Tipo 3, hemiglosectomías y hemiglosectomías extendidas, el método predilecto para su reconstrucción son colgajos cutáneos o fasciocutáneos libres microvasculares, como el $\operatorname{radial}^{7} \mathrm{y}$ el lateral de brazo $^{8}$ (Figura 8), debido a su fácil manipulación, plegamiento para dar la forma original ${ }^{9}$, mínimo abultamiento intraoral y fácil movilidad con el remanente lingual; permitiendo al paciente rápida rehabilitación fonatoria y de deglución ${ }^{10}$ (Figura 9).

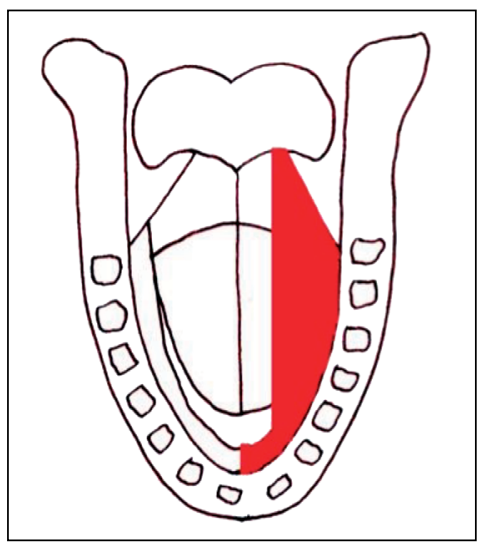

Figura 2. Hemiglosectomía subtotal o resección local ampliada.

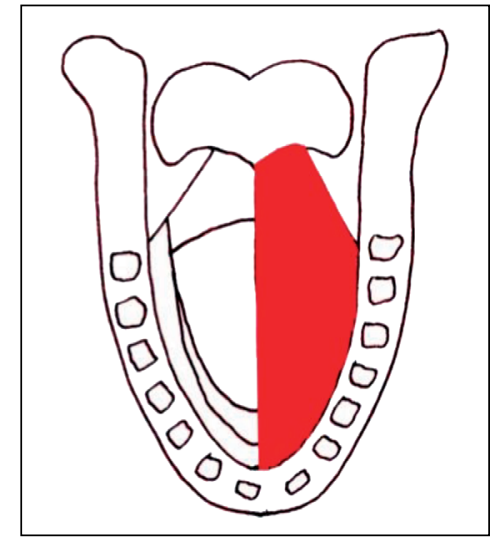

Figura 3. Hemiglosectomía.

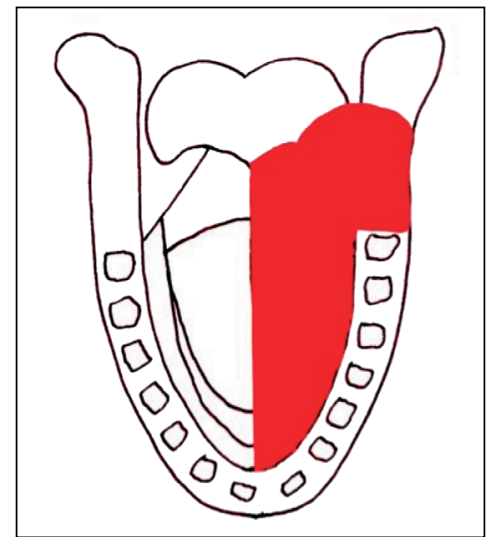

Figura 4. Hemiglosectomía extendida.

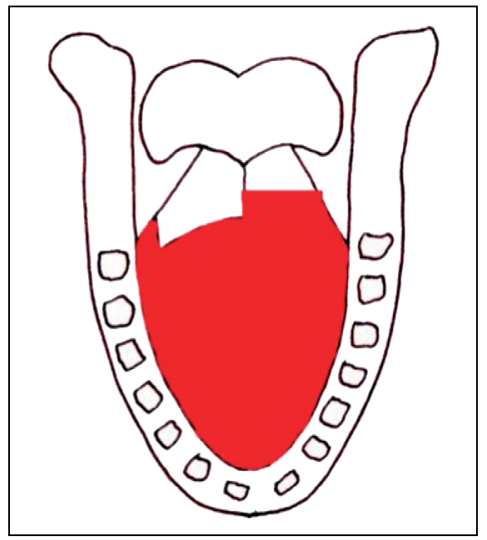

Figura 5. Glosectomía subtotal.

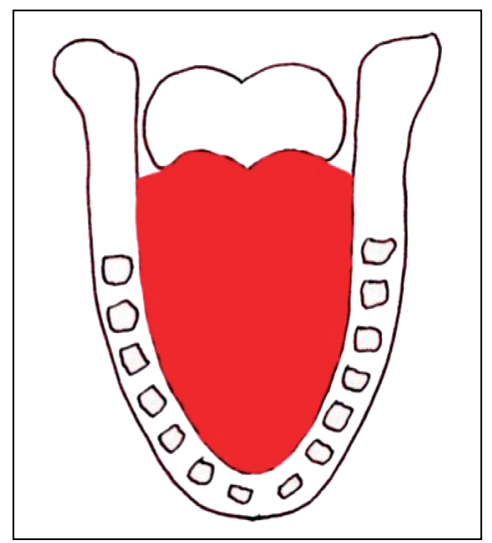

Figura 6. Glosectomía total. 


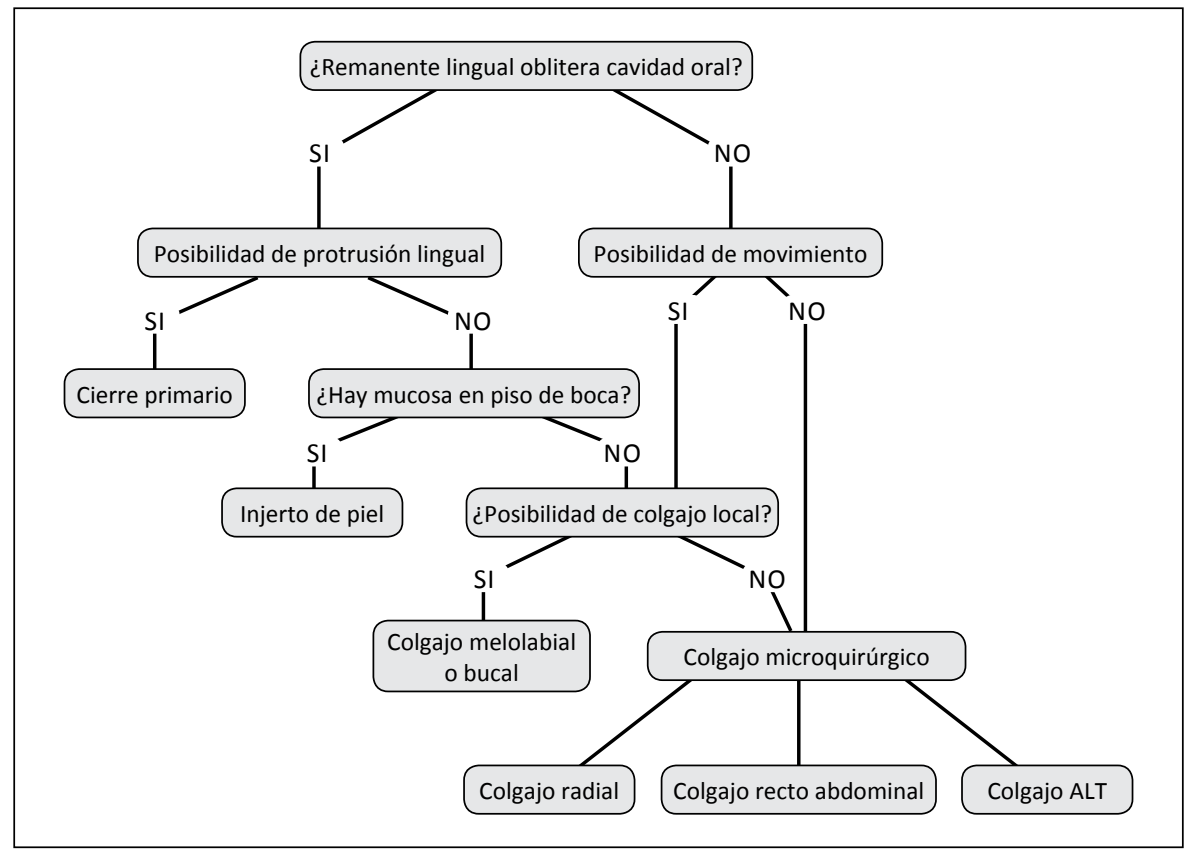

Figura 7. Algoritmo propuesto para reconstrucción lingual.

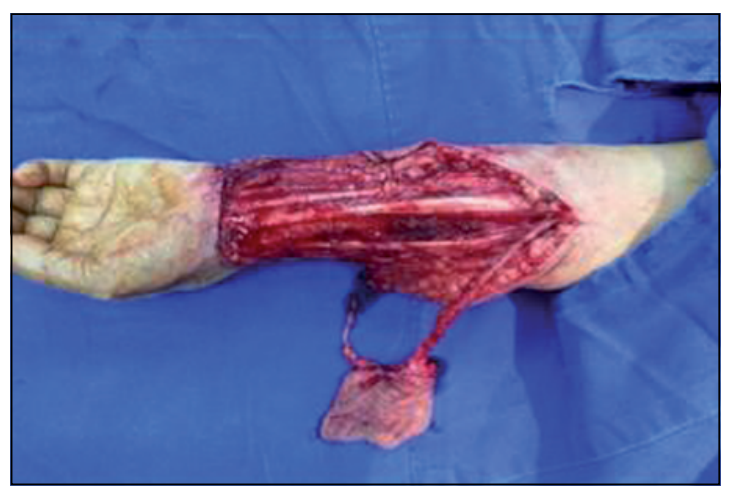

Figura 8. Disección de colgajo radial previa transferencia a cavidad oral.
En casos de defectos Tipo 4 y 5, debido a la necesidad de obliteración de la cavidad oral por parte del tejido transferido para devolver la deglución como objetivo principal, se prefieren colgajos bultosos ${ }^{11}$ como el colgajo musculocutáneo de recto abdominal (Figuras 10) y el colgajo fasciocutáneo anterolateral de muslo ${ }^{12,13}$ (Figura 11).

La complicación más importante de la reconstrucción lingual es la inhabilidad de hablar o comer a un nivel funcional, por lo que todos los esfuerzos del grupo multidisciplinario encargado deben ir dirigidos a evitarlo. Otras complicaciones que se presentan, como necrosis del remanente de lengua postresección, fístulas y atrofia de los colgajos, deben corregirse tempranamente ${ }^{14}$.

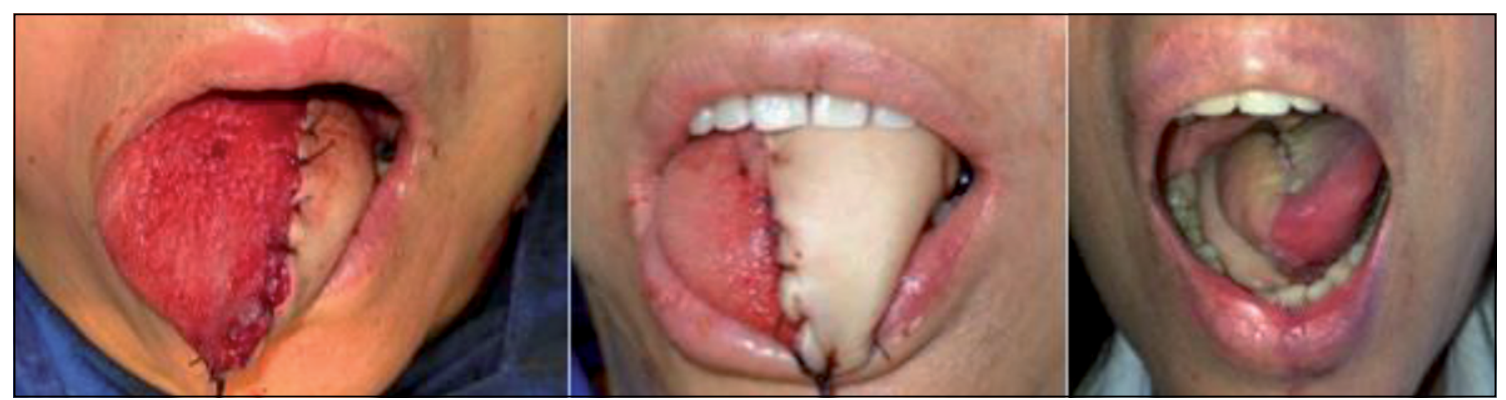

Figura 9. Reconstrucción de hemiglosectomía (defectos Tipo 2 y 3 ) con colgajo libre radial. Nótese el plegamiento del colgajo sobre sí mismo y en el piso de la lengua. 


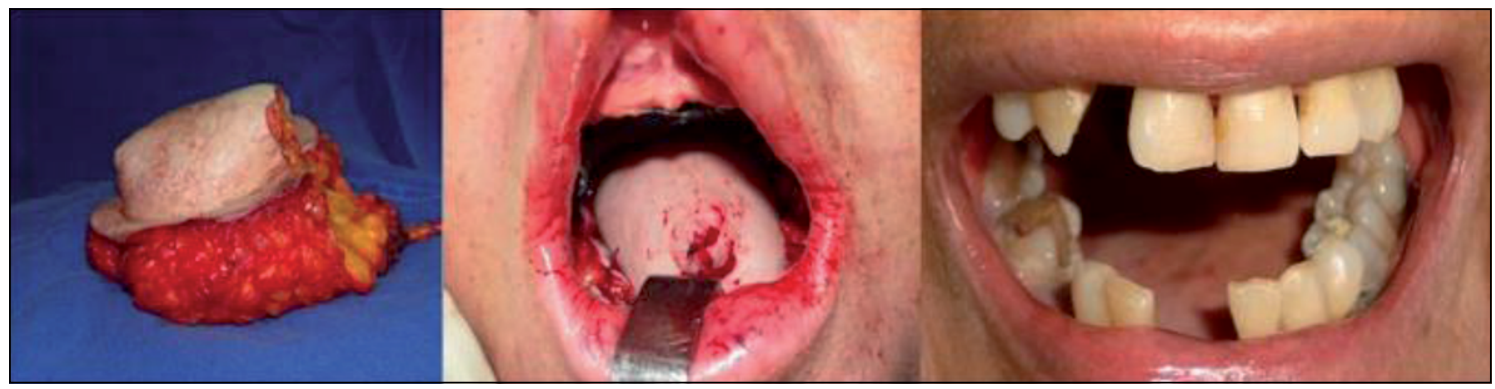

Figura 10. Modelamiento de colgajo libre musculocutáneo de recto abdominal, transferencia a cavidad oral y postoperatorio tardío.

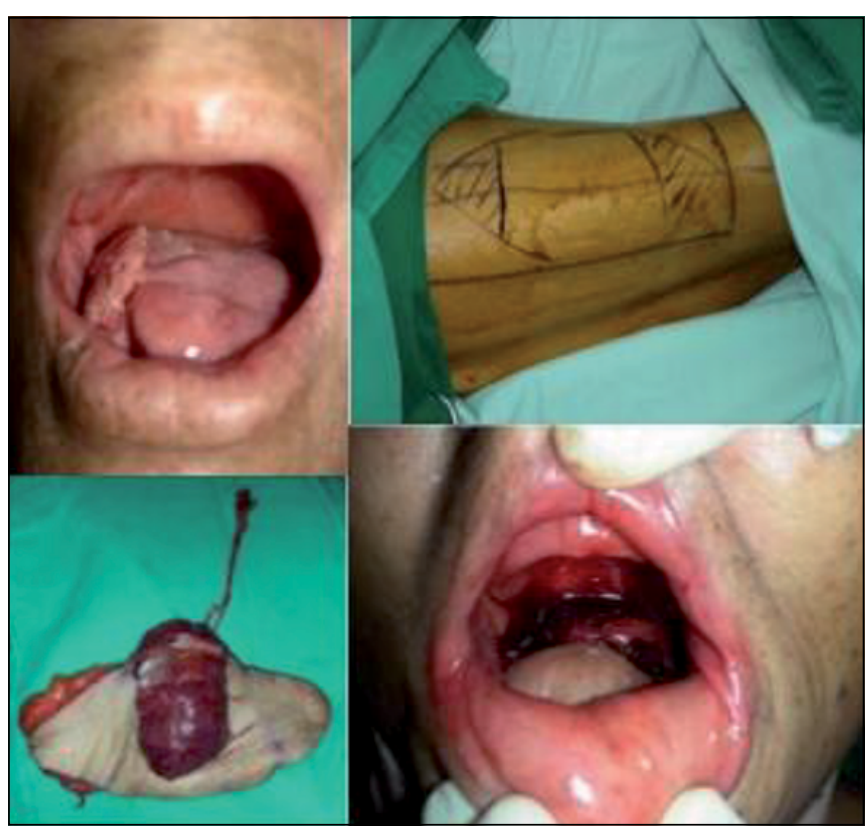

Figura 11. Carcinoma escamocelular de lengua y piso de boca, diseñando colgajo libre fasciocutáneo anterolateral de muslo con componente muscular.

\section{Discusión}

La lengua es fundamental para la deglución y la fonación por lo que su adecuada reconstrucción es de gran importancia para la calidad de vida de los pacientes sometidos a resecciones por tumores ${ }^{15}$. Las metas principales de la reconstrucción de lengua son el restablecimiento del lenguaje, la masticación y la deglución ${ }^{16}$, razón por la cual el tipo de reconstrucción a realizar debe ser cuidadosamente escogido.

Los colgajos presentan metaplasia completa en un período de dos a tres semanas, observándose un epitelio mucoso no secretor a nivel intraoral, el cual adopta la textura y la coloración de la lengua nativa, pero al mismo tiempo sufren atrofia especialmente si son sometidos a radioterapia, por lo cual los defectos deben ser sobrecorregidos para que finalmente per- manezca un adecuado volumen ${ }^{17}$.

Otros aspectos que se deben tener en cuenta al realizar la reconstrucción son la reposición anatómica del tejido lingual residual, preservando así movilidad y sensibilidad ${ }^{18}$, la resuspensión de la laringe para evitar broncoaspiración y una adecuada nutrición pre y postoperatoria, promoviendo una mejor cicatrización y pérdidas importantes de peso o desnutrición en los pacientes ${ }^{19}$.

Los resultados obtenidos con el esquema de tratamiento presentado han sido satisfactorios desde el punto de vista de recuperación de función deglutoria y fonatoria en defectos Tipo 1, 2 y 3 , y adecuada recuperación principalmente deglutoria para los defectos Tipo 4 y 5 con la asistencia temprana por parte de fonoaudiología, logrando buena readaptación a las actividades de la vida diaria de los pacientes tratados ${ }^{20}$. 


\section{Conclusión}

Los colgajos libres microvasculares han demostrado ser de gran utilidad para la reconstrucción de lengua con una adecuada recuperación de las funciones de la misma, sin prolongación de las estancias hospitalarias, con mínimo de complicaciones y con la ventaja de poder continuar su tratamiento oncológico de quimioterapia y radioterapia de su enfermedad de base. Esto es posible gracias a la adecuada tolerancia por parte de los tejidos transferidos a estos tratamientos adyuvantes, por lo que se hace indispensable evaluar el defecto y al paciente para escoger la reconstrucción más óptima. Al entender las funciones de la lengua, podemos tener claro cuales son los principios y objetivos de la reconstrucción, así como la continuidad del tratamiento que requiere este tipo de patologías $\mathrm{y}$, de este modo, poder ofrecer a nuestros pacientes el mejor tratamiento posible con reintegro social y laboral adecuados.

\section{Referencias}

1. Bell RB, Kademani D, Homer L, Dierks, EJ, Potter BE. Tongue cancer: Is there a difference in survival compared with other subsites in the oral cavity? Journal of Oral and Maxillofacial Surgery 2007;65:229-36.

2. Engel H, Huang JJ, Lin CY, Lam W, Kao HK, Gazyakan $\mathrm{E}$, et al. A strategic approach for tongue reconstruction to achieve predictable and improved functional and aesthetic outcomes. Plastic and Reconstructive Surgery 2010;126:1967-77.

3. Álvarez Martínez E, Preciado A, Montoya Fernández SA, Jiménez Gómez R, Posada A. Características clínico-histopatológicas del carcinoma escamocelular bucal, Colombia. Revista Cubana de Estomatología 2010;47:81-95.

4. Hanasono MM, Matros E, Disa JJ. Important aspects of head and neck reconstruction. Plastic and Reconstructive Surgery 2014;134:968e-980e.

5. Chepeha D. Tongue Reconstruction. In: Neligan P, Wei F-C, eds. Microsurgical Reconstruction of the Head and Neck. St Louis, MO: Quality Medical Publishing 2010:613-44.

6. Strauss RA, Kain NJ. Tongue Flaps. Oral and Maxillofacial Surgery Clinics of North America 2014;26:313-25.

7. Ko AB, Lavertu P, Rezaee RP. Double bilobed radial forearm free flap for anterior tongue and floor-of-mouth reconstruction. Ear Nose Throat J. 2010;89:177-9.

8. Thankappan K, Kuriakose MA, Chatni SS, Sharan R, Trivedi NP, Vijayaraghavan S, et al. Lateral arm free flap for oral tongue reconstruction: an analysis of surgical details, morbidity, and functional and aesthetic outcome. Annals of Plastic Surgery 2011;66:261-6.

9. Akashi M, Hashikawa K, Sakakibara A, Komori T, Terashi H. Long-term Follow-up Study of Radial Forearm Free Flap Reconstruction After Hemiglossectomy. Journal of Craniofacial Surgery 2015;26:44-7.

10. Vega C, León X, Cervelli D, Pons G, López S, Fernández $\mathrm{M}$, et al. Total or subtotal glossectomy with microsurgical reconstruction: functional and oncological results. Microsurgery 2011;31:517-23.

11. Gong ZJ, Zhang S, Ren ZH, Zhu ZF, Liu JB, Wu HJ. Application of anteromedial thigh flap for the reconstruction of oral and maxillofacial defects. Journal of Oral and Maxillofacial Surgery 2014;72:1212-25.

12. Jiang C, Guo F, Li N, Liu W, Su T, Chen X, et al. Multipaddled anterolateral thigh chimeric flap for reconstruction of complex defects in head and neck. PLoS One. 2014;9(9):e106326. doi: 10.1371/journal.pone.0106326. eCollection 2014.

13. Wang X, Yan G, Zhang G, Li J, Liu J, Zhang Y. Functional tongue reconstruction with the anterolateral thigh flap. World Journal of Surgical Oncology 2013;11:1-4.

14. Pierre CS, Dassonville O, Chamorey E, Poissonnet G, Riss JC, Ettaiche M, et al. Long-term functional outcomes and quality of life after oncologic surgery and microvascular reconstruction in patients with oral or oropharyngeal cancer. Acta Oto-laryngologica 2014; 134:1086-93.

15. Hartl DM, Dauchy S, Escande C, Bretagne E, Janot F, Kolb F. Quality of life after free-flap tongue reconstruction. The Journal of Laryngology \& Otology 2009;123:550-4.

16. Speksnijder CM, van der Bilt A, van der Glas HW, Koole R, Merkx MA. Tongue function in patients treated for malignancies in tongue and/or floor of mouth; a one year prospective study. International Journal of Oral and Maxillofacial Surgery 2011;40:1388-94.

17. Yun IS, Lee DW, Lee WJ, Lew DH, Choi EC, Rah DK. Correlation of neotongue volume changes with functional outcomes after long-term follow-up of total glossectomy. Journal of Craniofacial Surgery 2010;21:111-6.

18. Guerin-Lebailly C, Mallet Y, Lambour V, Fournier C, El Bedoui S, Van JT, et al. Functional and sensitive outcomes after tongue reconstruction: About a series of 30 patients. Oral Oncology 2012;48:272-7.

19. Brown L, Rieger JM, Harris J, Seikaly H. A longitudinal study of functional outcomes after surgical resection and microvascular reconstruction for oral cancer: tongue mobility and swallowing function. Journal of Oral and Maxillofacial Surgery 2010;68:2690-700.

20. Canis M, Weiss BG, Ihler F, Hummers-Pradier E, Matthias C, Wolff HA. Quality of life in patients after resection of pT3 lateral tongue carcinoma: Microvascular reconstruction vs primary closure. Head Neck. 2014 Sep 15. doi: 10.1002/hed.23862. [Epub ahead of print]. 